CLINICAL STUDY

\title{
The incidence of second primary tumors in thyroid cancer patients is increased, but not related to treatment of thyroid cancer
}

\author{
Robbert B T Verkooijen, Jan W A Smit, Johannes A Romijn and Marcel P M Stokkel \\ Department of Nuclear Medicine, Leiden University Medical Center, PO Box 9600, 2300 RC Leiden, The Netherlands \\ (Correspondence should be addressed to M P M Stokkel; Email: m.p.m.stokkel@lumc.nl)
}

\begin{abstract}
Objective: The aim of the present study is to assess the prevalence of second primary tumors in patients treated for thyroid cancer. Furthermore, we wanted to assess the standardized risk rates for all second primary tumors, but especially for breast cancer, as data in the literature indicate an excessive risk in differentiated thyroid cancer (DTC) patients for this tumor.

Materials and methods: We included consecutive patients, who received ablation treatment with I-131 at the Leiden University Medical Center between January 1985 and December $1999(n=282)$. The mean period of follow-up was $10.6 \pm 4.1$ years.

Results: Thirty-five of the 282 patients (12.4\%) had a second primary tumor (SPT), either preceding or following the diagnosis of thyroid cancer. Five other patients had three primary tumors, including DTC. As a result, 40 additional tumors were found in this group, revealing an overall prevalence of $14.2 \%$. Twenty tumors $(7.1 \%)$ preceded the thyroid cancer with a mean interval of 5.7 years (range: 0.5-22.0 years), whereas 20 tumors $(7.1 \%$ ) occurred after this tumor with a mean interval of 6.7 years (range: $1.0-15.0$ years). In 13 female patients, breast cancer was found as SPT. The standardized incidence rate (SIR) for all cancers after the diagnosis of DTC in this study population was not increased (1.13; confidence interval (CI): 0.68-1.69). However, we found an increased SIR of 2.26 (CI: 1.60-3.03) for all cancers either following or preceding DTC, which is mainly caused by a SIR of 3.95 (CI: 2.06-6.45) for breast cancer. Conclusion: Patients with DTC have an overall increased standardized incidence rate for second primary tumors, but not for second primary tumors following I-131 therapy. These findings suggest a common etiologic and/or genetic mechanism instead of a causal relation.
\end{abstract}

European Journal of Endocrinology 155 801-806

\section{Introduction}

Differentiated thyroid cancer (DTC) is the most frequent endocrine gland malignancy accounting for approximately $0.5-1.5 \%$ of all malignancies. The overall survival rate is high and currently even close to $99 \%$ in papillary carcinomas $(10,22)$. The standard treatment for DTC is a (near-) total thyroidectomy followed by ablation therapy using high doses of radioiodine-131 (I-131), except in small papillary thyroid cancer or minimal invasive follicular carcinomas. Although many reports have focused on the relationship between I-131 administration and the occurrence of second primary tumors, some studies found (e.g. 19) a relationship between I-131 administration and the occurrence of bone and soft tissue, colorectal and salivary gland cancers. Other studies reported an overall increased incidence of breast and kidney cancer among women treated for thyroid cancer, but this was not related to the exposure of I-131 $(6,20,23)$.
The aim of the present study is to assess the prevalence of second primary tumors in patients treated for thyroid cancer using an uptake-related ablation strategy. Furthermore, we wanted to assess the standardized risk rates for all second primary tumors, but especially for breast cancer, as data in literature indicate an excessive risk in DTC patients for this tumor.

\section{Materials and methods}

We included consecutive patients, who received ablation treatment with I-131 at the Leiden University Medical Center (LUMC) between January 1985 and December $1999(n=282)$ for DTC. This period was selected, as since 1985 standardized ablation doses were given. The mean period of follow-up was $10.6 \pm 4$.1 years. From the medical files, age, gender, histopathological data, ablation dose, and subsequent I-131 treatments were recorded. Furthermore, data with respect to second primary tumors were gained, including site and interval. 
In addition, malignancies preceding the diagnosis of DTC were recorded. However, in situ and basal cell carcinomas were excluded. All information with respect to malignancies was obtained from the medical records as well as from the central hospital registration system for malignancies. This oncology documentation system, the ONCDOC, gathers information about tumors, followup, recurrences, second primaries, therapeutic interventions, survival, etc. To define the extent of thyroid carcinomas, the sixth edition of the tumour, node, metastasis (TNM) staging was used.

The ratio of the observed $(\mathrm{O})$ numbers to the expected (E) number (i.e. the standardized incidence ratio; SIR) was computed using cancer incidence data from the general population. These specific incidence rates were derived from the Dutch national cancer registry (www.ikcnet.nl) by gender, 5- and 15-year age groups and were multiplied by the accumulated person-years in the study cohort to estimate the expected number of cancer cases.

\section{Radioiodine treatment}

The mean interval between a thyroidectomy and the ablative treatment in the present population was $5.4 \pm$ 4.0 weeks. During this interval, patients did not receive any thyroid hormone supplementation with the aim of increasing the thyroid-stimulating hormone levels. All patients treated from 1992 were put on a low-iodine diet that started 4 days prior to ablation to facilitate an optimal uptake of I-131 in the thyroid remnant. Before 1992, lowiodine diets were not routinely prescribed (15).

The ablation dose of I-131 was determined by the 24-h I-131 pre-treatment uptake value in the neck region. Therefore, patients received $40 \mathrm{MBq}$ of I-131 orally, which was followed by planar scintigraphy of the neck region $24 \mathrm{~h}$ later. Uptake values less than 5\%, between 5 and $10 \%$, and values more than $10 \%$ were followed by 2800, 1850, and $1100 \mathrm{MBq}$ of I-131 in one dose respectively. The rationale of this uptake-related approach is to avoid unnecessary exposure and local radioiodine side effects $(25)$.

\section{Results}

\section{Clinical characteristics}

Overall, we treated 282 patients, 219 female, and 63 male (age $47.5 \pm 17.5$ years). During long-term followup, 64 patients died. The mean period of follow-up was $10.6 \pm 4.1$ years. Tumor characteristics are presented in Table 1, demonstrating that T2, NO tumor stage and papillary thyroid cancer were most frequently observed.

The mean 24-h I-131 uptake was $6.3 \pm 7.7 \%$. The mean ablation dose given was $2616 \pm 1103 \mathrm{MBq}$ of I-131, whereas the overall cumulative dose given ranged from $1100 \mathrm{MBq}$ up to $49 \mathrm{GBq}$ (mean: $7657 \mathrm{MBq})$ of I-131.
Table 1 Tumor and treatment characteristics.

\begin{tabular}{lc}
\hline Characteristics & $\begin{array}{c}\text { Results } \\
(n=282)\end{array}$ \\
\hline Histology & \\
Papillary carcinoma & 156 \\
Follicular carcinoma & 51 \\
Follicular variant of papillary & 57 \\
carcinoma & \\
Hürthle cell carcinoma & 17 \\
Not further specified & 1 \\
T-stage & \\
1 & 20 \\
2 & 153 \\
3 & 45 \\
4 & 61 \\
X & 3 \\
N-stage & \\
0 & 209 \\
1 & 70 \\
X & 3 \\
M-stage & \\
0 & 232 \\
1 & 50 \\
24 h I-131 uptake (\%) & $6.3 \pm 7.7$ \\
Mean I-131 ablation does (MBq) (s.D.) & $2616 \pm 1103$ \\
Mean cumulative I-131 treatment dose & $7657(1100-49000)$ \\
(MBq) (range) & \\
&
\end{tabular}

T-stage, tumor stage; $\mathrm{N}$-stage, node stage; $\mathrm{M}$-stage, metastasis stage.

\section{Additional tumors}

Overall, 35 patients (12.4\%) had a second primary tumor either preceding or following the thyroid cancer (see Table 2). Five other patients had three primary tumors, including DTC, according to the medical files. As a result, in 40 patients additional tumors were found in this group, revealing an overall prevalence of $14.2 \%$ : 20 tumors $(7.1 \%)$ preceded the thyroid cancer with a mean interval of 5.7 years (range: $0.5-22.0$ years) and 20 tumors $(7.1 \%)$ occurred after this tumor with a mean interval of 6.7 years (range: $1.0-15.0$ years). In 13 out of 219 female patients $(5.9 \%)$, breast cancer was documented. Regarding the mean period of follow-up of 10.6 years, we found an overall prevalence of $0.67 \%$ of second primaries per year following DTC and $0.5 \%$ per year when excluding breast cancer. Finally, the total follow-up years, defined as the mean period of follow-up times the number of patients studied, was 2989 years. Regarding this parameter, it resulted in an overall prevalence for second primaries following DTC of 0.0024 and $0.0018 \%$ per person follow-up year with and without breast cancer respectively.

In Table 3, the standardized incidence ratios are described. The SIR of second primary tumor (SPT), following treatment of thyroid cancer was not increased (1.13; 95\% CI: 0.68-1.69). However, the SIR of all SPT, i.e. including all cancers occurring before as well after the diagnosis of DTC, was increased (2.26; 95\% confidence interval (CI): 1.60-3.03). This was mainly due to an increased incidence of breast cancer (SIR 3.29; 95\% CI: 2.06-6.45). 
Table 2 Locations of second primary tumors in differentiated thyroid cancer (DTC) patients.

\begin{tabular}{|c|c|c|c|c|c|c|c|}
\hline $\begin{array}{l}\text { Tumors preceding } \\
\text { DTC }\end{array}$ & $n$ & $\begin{array}{l}\text { Mean cumulative } \\
\text { I-131 activity (MBq) }\end{array}$ & $\begin{array}{l}\text { Mean interval } \\
\text { (years) (range) }\end{array}$ & $\begin{array}{c}\text { Tumors } \\
\text { following DTC }\end{array}$ & $n$ & $\begin{array}{l}\text { Mean cumulative } \\
\text { I-131 activity }(\mathrm{MBq})\end{array}$ & $\begin{array}{c}\text { Mean interval } \\
\text { (years) } \\
\text { (range) }\end{array}$ \\
\hline Melanoma & 2 & 22400 & $13(4-22)$ & Melanoma & 1 & 1850 & 1 \\
\hline Breast & 8 & 4900 & $4.3(2-10)$ & Breast & 5 & 9000 & $6.6(2-11)$ \\
\hline Colon & 2 & 9100 & $3.3(0.5-6)$ & Cervix uteri & 2 & 5050 & $4(1-7)$ \\
\hline Lung & 2 & 8400 & $2.3(0.5-4)$ & Ovary & 2 & 2400 & $8.5(2-15)$ \\
\hline Adrenal & 1 & 8900 & 15 & Endometrium & 1 & 16512 & 7 \\
\hline Salivary gland & 1 & 2800 & 17 & Pancreas & 2 & 2800 & $7(3-11)$ \\
\hline Grawitz tumor & 1 & 7400 & 7 & Bladder & 1 & 2800 & 3 \\
\hline Stomach & 1 & 24735 & 2 & Lung & 1 & 2800 & 14 \\
\hline Prostate & 1 & 26740 & 2 & Prostate & 1 & 9400 & 1 \\
\hline \multirow[t]{4}{*}{ Lymphoma } & 1 & 15725 & 0.5 & Hepatocellular & 1 & 15400 & 2 \\
\hline & & & & Grawitz & 1 & 21100 & 14 \\
\hline & & & & Leukemia & 1 & 2800 & 8 \\
\hline & & & & Lymphoma & 1 & 2800 & 12 \\
\hline Overall & 20 & & $5.7(0.5-22)$ & Overall & 20 & & $6.7(1-15)$ \\
\hline
\end{tabular}

SPT, second primary tumor.

\section{Discussion}

Patients with DTC have a very good prognosis, which is achieved by surgery and postoperative ablation with I-131 therapy. Our data indicate that this treatment is not associated with increased incidence of malignancies following treatment of DTC. However, the data indicate that there is an increased incidence of second primary tumors in thyroid cancer patients if we consider their lifetime risks for cancer, especially of breast cancer.

Regarding the incidence and prevalence of SPT in general, an 1/3 rule has been hypothesized, which means that approximately $33 \%$ of the patients with a malignancy will develop an SPT, 33\% of these patients with an SPT a third malignancy, and so on (17). A welldescribed phenomenon, in this respect, is the field cancerization concept, which states that organ systems exposed to the same carcinogenic agents have a greater chance of transforming into a malignancy. One of the most striking examples of this concept is the combination of head and neck cancer with lung malignancies (8). Another explanation for SPT may be the presence of a genetic base for multiorgan cancer (7). These authors studied a multiorgan cancer susceptibility gene, the CHEK2 protein, in 4008 cancer cases and 4000 controls. This CHEK2 protein participates in the DNA damage response in many cell types. The missense variant I157T was associated with an increased risk of breast cancer, colon cancer, kidney cancer, prostate cancer, and thyroid carcinoma. Therefore, the authors concluded that cancers associated with mutations of the CHEK2 gene might be much greater than previously thought.

In some studies, an increased incidence of breast carcinoma has been suggested in women with thyroid carcinoma. Vassilopoulou-Sellin et al. (24) published one of the first studies on this, including 41686 patients with breast carcinoma and 3662 with thyroid carcinoma, which were retrospectively analyzed. Among 18931 women with a diagnosis of breast carcinoma, 11 developed DTC more than 2 years after the initial diagnosis of breast cancer. The observed incidence of DTC was not different from that in a similar group of women without breast cancer. Among 1013

Table 3 Ratio of observed to expected second primary tumors (SPT) following differentiated thyroid cancer (DTC) and both preceding and following differentiated DTC.

\begin{tabular}{|c|c|c|c|c|c|c|c|c|}
\hline \multirow[b]{2}{*}{ Site of SPT } & \multicolumn{4}{|c|}{ SPT following DTC } & \multicolumn{4}{|c|}{ All SPT prior to and/or following DTC } \\
\hline & $\mathrm{O}_{\mathrm{i}}$ & $\mathrm{E}_{\mathrm{i}}$ & SIR & $95 \% \mathrm{Cl}$ & $\mathrm{O}_{\mathrm{i}}$ & $E_{i}$ & SIR & $95 \% \mathrm{Cl}$ \\
\hline Head and neck & - & 1.16 & - & - & 1 & 1.16 & 0.86 & $0.01-3.45$ \\
\hline Lung & 1 & 6.47 & 0.15 & $0.01-0.62$ & 3 & 6.47 & 0.46 & $0.08-1.15$ \\
\hline Breast & 5 & 3.29 & 1.52 & $0.46-3.18$ & 13 & 3.29 & 3.95 & $2.06-6.45$ \\
\hline Digestive tract & 3 & 9.12 & 0.33 & $0.06-0.82$ & 6 & 9.12 & 0.66 & $0.23-1.30$ \\
\hline Female genitourinary tract & 7 & 4.35 & 1.61 & $0.62-3.06$ & 9 & 4.35 & 2.07 & $0.92-3.68$ \\
\hline Prostate & 1 & 1.59 & 0.63 & $0.01-2.52$ & 2 & 1.59 & 1.26 & $0.11-3.67$ \\
\hline Melanoma & 1 & 1.05 & 0.95 & $0.01-3.81$ & 3 & 1.05 & 2.86 & $0.51-7.11$ \\
\hline Others & 2 & - & - & - & 3 & - & - & - \\
\hline Total & 20 & 17.70 & 1.13 & $0.68-1.69$ & 40 & 17.70 & 2.26 & $1.60-3.03$ \\
\hline
\end{tabular}

$\mathrm{O}_{i}$, observed number of second primary tumors; $\mathrm{E}_{\mathrm{i}}$, expected number of second primary tumors; SIR, standardized incidence ratio; $95 \% \mathrm{Cl}$, $95 \%$ confidence interval. 
women with a diagnosis of thyroid carcinoma, 24 developed breast cancer more than 2 years after the primary tumor. In that group, the observed incidence of breast cancer in women of age 40-49 years was significantly higher than the expected incidence for women in the same age group. However, in a study by Sadetzki et al. (20), opposite results were observed. This group found an overall significant elevated risk for DTC following breast cancer in 49207 female studied. The observed number of cases of DTC after breast cancer was 59 , revealing a standardized incidence ratio of 1.34 (95\% CI: 1.03-1.72), as the expected number of cases was 43 . This ratio was 1.07 for breast cancer following DTC. In addition, the authors concluded that considering the long latency required for carcinogenesis and the excess risk of SPT soon after the index tumor argues against the hypothesis of primary tumor treatment as initiator. The authors suggested that early exposure to common risk factors or genetic susceptibility for both malignancies to be plausible. Cengiz et al. (6) observed an increased frequency of thyroid pathology in breast cancer patients compared with controls, but it has to be realized that the high percentage of thyroid disorders in that study might be related to the fact that Turkey is an endemic region for thyroid diseases. In a study by Simon et al. (23), only women with a history of thyroid cancer were found to have an increased risk, but this was restricted to parous women. The overall odds ratio in this respect was 3.5 (95\% CI: 1.5-8.1). However, they concluded that, despite the increased incidence, there was no association between thyroid cancer treatment and the risk on breast cancer. Finally, Pal et al. (14) performed a study on double primary cancers of the breast and thyroid in women and assessed a molecular and genetic base.

Their data did not support the hypothesis that a significant proportion of double primary cancers are due to hereditary factors. In the present study, we found a standardized incidence ratio of 1.52 (95\% CI: 0.463.18) for breast cancer comparable with the data published by Berthe et al. (4). Moreover, an increased overall incidence (the SIR increases up to 3.95 (95\% CI: 2.06-6.45)) for breast cancer in thyroid cancer patients was observed, when taking also the tumors preceding DTC into account. Based on data in literature, it is highly unlikely that ionizing radiation is involved in the genesis of breast cancer in DTC patients. Genetic predisposition and probably environmental factors seem to be a better explanation for the coincidence, as at least half of the breast tumors appear before treatment with I-131 for DTC.

A relation between the total dose of I-131 and SPT has been reported for solid cancers and leukemias with increasing cumulative activity of the dose administered (19). The question remains whether this is related to the administration of I-131 solely or also to an increased organ sensitivity for carcinogens in general. Hemminki et al. (11) described an increased risk of cancer by site and histopathology, in which familial risks with SIRs over 4.0 were found for ovary cancer, thyroid cancer, and clear cell carcinoma. For this purpose, they used a nationwide Swedish Family cancer database on 10.2 million individuals and 1 million tumors to calculate SIRs for familial cancers. Their results called for a closer description of tumor-susceptibility genes. It was the group of Fisher, who firstly studied the expression of five gene family members in relation to different cancers (9). It is beyond the scope of this article to report on these findings in detail, but an important finding was the observation of significantly elevated expression levels of osteopontin in cancer of the breast, ovary, uterus, and thyroid compared with normal tissue levels. In this study, the relative mRNA expression units quantified using ImageQuant for normal tissue and these tumor subtypes were as follows: 18.000 and 54.000 respectively, for normal breast tissue and breast cancer; 14.000 and 70.000 respectively, for normal tissue of the uterus and cancer of the uterus; 20.000 and 126.000 respectively, for normal ovary tissue and ovary cancer; 4.000 and 16.000 respectively, for thyroid tissue and thyroid cancer. Especially, the relation between these glycoproteins and matrix metalloproteinases, critical for the development of wound healing and cancer progression, is suggestive for a common genetic base. Ronckers et al. recently published a large report on thyroid cancer and multiple primary tumors (18). They studied the risk of thyroid cancer after an earlier primary cancer, as well as the risk of developing multiple cancers after an earlier thyroid cancer in the US, using the cancer registry program of more than 2 million patients. They observed an absolute excess risk for all cancers following DTC of 7.64, which is mainly based on an increased risk for female breast, prostate, and kidney cancer. Breast cancer contributed $36 \%$ of all second cancers after DTC. Patients $<40$ years of age at diagnosis of DTC had a 39\% increased risk of a second cancer, whereas for older patients this was $6 \%$. On the other hand, breast cancer patients had a 1.3-fold risk of developing DTC. Several other cancer sites showed significantly increased risks in both directions, including salivary glands, prostate, breast, kidney, scrotum, brain, and leukemia. Although I-131 therapy may play a role in the induction of some of these tumors, the high incidence of tumors preceding DTC suggest etiologic similarities. Finally, Adjadj et al. (1) reported comparable results obtained from 2365 women who were treated for thyroid carcinoma. In this series, 48 women developed subsequent breast carcinoma, revealing an overall SIR of 1.3, but even up to 2.5 for patients treated at an age ranging from 4 to 30 years. The authors did not find a correlation between the high SIR and radiation treatment with I-131, but the co-occurrence may be related to a genetic deregulation, as the H23 gene, which is overexpressed in both DTC and breast cancer. It is still not clear why the gene coding for $\mathrm{H} 23$ antigen mRNA is overexpressed in these cancers. As one 
of its forms is probably a transmembrane receptor-like protein, it may be an element of signal transduction and, therefore, reflect an involvement in cell growth $(26,27)$.

Recent reports have focused on malignancies within the scope of certain cancer syndromes. Mutations in MLH1 and MSH2, genes coding for mismatch repair enzymes that are involved in the repair of DNA replication errors, are associated not only with an increased risk of hereditary nonpolyposis colorectal cancer, but also with other malignancies, such as cancer of the stomach, urinary tract, and thyroid. (5, $12,13)$. It may be an explanation for some of the present findings related to thyroid cancer. However, a correlation between MSH2 mutations and breast cancer as SPT in thyroid cancer patients, or vice versa, has never been described. Another gene that is associated with cancer syndromes is the PTEN gene, which is located on chromosome 10q23. This phosphatase suppressor tumor gene downregulates cell survival through apoptosis and/or G1 cell cycle arrest. It is characterized by an increased risk of benign and malignant tumors of the breast, thyroid, and endothelium $(2,3,16)$. Consequently, these studies stress the need to better screen for clonal PTEN rearrangements and gene mutations, especially in DTC patients presenting with second primaries of the breast.

Recently, Sandeep et al. published a large multinational record linkage study on second primary cancers in thyroid cancer patients (21). The study was conducted at 13 population-based cancer registries in Europe, Canada, Australia, and Singapore and included a cohort of 39.002 patients with primary thyroid cancer. During an observation period of 356.035 person-year, 2821 second primary tumors were observed resulting in an overall SIR of 1.31 (CI: 1.261.36). Remarkable increased incidence rates were found for cancer of the oral cavity (SIR: 1.43), small intestine (SIR: 2.11), bone (SIR: 3.62), soft tissue sarcoma (SIR: 3.63), kidney (SIR: 2.33), endocrine glands (SIR: 6.75), lymphoma (SIR: 1.68), and leukemias (SIR: 2.26). The SIR for breast cancer was 1.31 , which is definitely lower than present findings. However, this discrepancy can be fully ascribed to the differences in number of patients studied and in period of follow-up. The group of Sandeep et al. also found and increased incidence of second primary thyroid cancer after cancers of the lung, larynx, esophagus, and salivary gland. Although a relation between initial treatment, such as radiotherapy, for the index tumor and subsequent thyroid cancer may be suggested, the short interval and age of initial treatment do not support this hypothesis. Nevertheless, we fully ascribe their conclusion that clinicians should maintain a high index of suspicion, both for second primaries following treatment for thyroid cancer and for cancer of the thyroid as SPT.

A limitation of our study is the small number of patients included in the database. Patients who received ablation treatment with I-131 at the LUMC between January 1985 and December 1999 were selected for analysis. The mean period of follow-up is more than 10 years, which gives a good estimate of the relative risk and its relation to the induction of SPT. In most other series on this subject, the interval between therapy and the occurrence of a therapy-related SPT is up to 5 years.

In conclusion, we studied the incidence of second primary tumors in relation to DTC and found an overall increased SIR for all cancers, but especially for breast cancer. Despite the relatively small number of patients studied, the present results are highly suggestive for a common etiologic and/or genetic mechanism. In this respect, further study is required, but in agreement with recently published data by others, close clinical followup of thyroid cancer patients may be recommended for the early diagnosis of second primary tumors.

\section{References}

1 Adjadj E, Rubino E, Shamdaldim A, le Monique G, Schlumberger M \& de Vathaire F. The risk of multiple primary breast and thyroid carcinomas: role of radiation dose. Cancer 200398 1309-1317.

2 Agrawal S \& Eng C. Differential expression of novel naturally occurring splice variants of PTEN and their functional consequences in Cowden syndrome and sporadic breast cancer. Human Molecular Genetics 200615 777-787.

3 Bau MG, Arisio R, Cristini G, Bertone E \& Campgrande M. Screening-detected breast carcinoma in a patient with Cowden syndrome. Breast 200413 239-241.

4 Berthe E, Henry-Amar M, Michel J-J, Rame J-P, Berthet P, Babin E, Icard P, Samama G, Galateau-Salle F, Mahoudeau J \& Bardet S. Risk of second primary cancer following differentiated thyroid cancer. European Journal of Nuclear Medicine and Molecular Imaging 200431 685-691.

5 Broaddus RR, Lynch PM, Lu KH, Luthra R \& Michelson SJ. Unusual tumors associated with the hereditary nonpolyposis colorectal cancer syndrome. Modern Pathology 2004 17 981-989.

6 Cengiz O, Bozkurt B, Unal B, Yildrim O, Karaeyoglu M, Eroglu A, Kocer B \& Ulas M. The relationship between prognostic factors of breast cancer and thyroid disorders in Turkish women. Journal of Surgical Oncology 200487 19-25.

7 Cybulski C, Gorski B, Huzarski T, Masojc B, Mierzejewski M, Debniak T, Teodorczyk U, Byrski T, Gronwald J, Matyjasik J, Zlowocka E, Lenner M, Grabowska E, Nej K, Castaneda J, Medrek K, Szymanska A, Szymanska J, Kurzawski G, Suchy J, Oszurek O, Witek A, Narond SA \& Lubinski J. CHEK2 is a multiorgan cancer susceptibility gene. American Journal of Human Genetics 200475 1131-1135.

8 Duchateau CSJ \& Stokkel MPM. Second primary tumors involving non-small cell lung cancer: prevalence and its influence on survival. Chest 2005127 1152-1158.

9 Fisher LW, Jain A, Tayback M \& Fedarko SN. Small integrin binding ligand $\mathrm{N}$-linked glycoproteins gene family expression in different cancers. Clinical Cancer Research 200410 8501-8511.

10 Gilliland FD, Hunt WC, Morris DM \& Key CR. Prognostic factors in thyroid carcinoma. A population-based study of 15698 cases from the surveillance, epidemiology and end results (SEER) program 1973-1991. Cancer 199779 564-573.

11 Hemminki K \& Li X. Familial risk of cancer by site and histopathology. International Journal of Cancer 2003103 105-109.

12 Hemminki K \& Chen B. Familial association of colorectal adenocarcinoma with cancers at other sites. European Journal of Cancer $2004402480-2487$. 
13 Levene S, Scott G, Price P, Sandersom J, Evans H, Taylor C, Bass S, Lewis C \& Hodgson S. Does the occurrence of certain rare cancers indicate and inherited cancer susceptibility? Familial Cancer 2003 2 15-25.

14 Pal T, Hamel N, Vespirini D, Sanders K, Mitchell M, Quercia N, Ng Cheong N, Murray A, Foulkes WD \& Narod SA. Double primary cancers of the breast and thyroid in women: molecular analysis and genetic implications. Familial Cancer 20011 17-24.

15 Pluijmen MJH, Eustatia-Rutten C, Goslings B, Stokkel MP, Pereira Arias AM, Diamant M, Romijn JA \& Smit JWA. Effects of low-iodide diet on postsurgical radioiodide ablation therapy in patients with differentiated thyroid carcinoma. Clinical Endocrinology $2003 \mathbf{5 8}$ $428-435$.

16 Puxeddu E, Zhao G, Stringer JR, Medvedovic M, Moretti S \& Fagin JA. Characterization of novel non-clonal intrachromosomal rearrangements between the $\mathrm{H} 4$ and PTEN genes (H4/PTEN) in human thyroid cell lines and papillary thyroid cancer specimens. Mutation Research $2005 \mathbf{5 7 0}$ 17-32.

17 Reingold SR, Neugut AI \& Meadows AT. Second cancers: incidence, risk factors and management. In Cancer Medicine, pp 2399-2406. Eds JF Holland, E Frei, RE Pollock, RR Weichselbaum, RC Bast \& DW Kufe : Hamilton, ON, Canada, 2000.

18 Ronckers CM. Thyroid cancer and multiple primary tumors in the SEER cancer registries. International Journal of Cancer 2005117 281-288.

19 Rubino C, de Vathaire F, Dottorini ME, Hall P, Schvartz C, Couette JE, Dondon MG, Abbas MT, Langlois C \& Schlumberger M. Second primary malignancies in thyroid cancer patients. British Journal of Cancer 200389 1638-1644.

20 Sadetzki S, Calderon-Margalit R, Peretz C, Novikov I, Barchana M \& Papa MZ. Second primary breast cancer and thyroid disorders. Cancers Causes and Control 200214 367-375.

21 Sandeep TC, Strachan MWJ, Reynolds RM, Brewster DH, Scelo G, Pukkula E, Hemminki K, Anderson A, Tracey E, Friis S,
McBride ML, Kee-Seng C, Pompe-Kirn V, Kliewer EV, Tonita JM, Jonasson JG, Martos C, Bofetta P \& Brennan P. Second primary cancers in thyroid cancer patients: a multinational record linkage study. Journal of Clinical Endocrinology and Metabolism 200691 1819-1825.

22 Schlumberger MJ. Papillary and follicular thyroid carcinoma. New England Journal of Medicine 1998338 297-306.

23 Simon M, Tang M-TC, Berstein L, Norman SA, Weiss L, Burkman RT, Daling JR, Deapen D, Folger SG, Malone K, Marchbanks PA, McDonald JA, Strom BL, Wilson HG \& Spirtas R. Do thyroid disorders increase the risk of breast cancer. Cancer Epidemiology, Biomarkers and Prevention 2002 11 1574-1578.

24 Vassilopoulou-Sellin R, Palmer L, Palmer S, Taylor S \& Cooksley CS. Incidence of breast carcinoma in women with thyroid carcinoma. Cancer 199985 696-705.

25 Verkooijen RBT, Stokkel MPM, Smit JWA \& Pauwels EKJ. Radioiodine-131 in differentiated thyroid cancer: a retrospective analysis of an uptake-related ablation strategy. European Journal of Nuclear Medicine and Molecular Imaging 200431 499-506.

26 Weiss M, Zaretsky J, Zimlichman R, Smorodinsky N, Dion AS, Keydar I \& Wreschner DH. Expression of a gene coding for breast tumor-associated antigen in thyroid papillary carcinoma. Cancer Letters 199158 125-130.

27 Zaretsky JZ, Weiss M, Tsarfaty I, Hareuveni M, Wreschner DH \& Keydar I. Expression of genes coding for pS2, c-erbB2, estrogen receptor and the H23 breast tumor-associated antigen. FEBS Letters 1990253 46-50.

Received 14 June 2006

Accepted 19 September 2006 